\title{
Cascade of granular flows for characterizing segregation
}

\author{
G. Lumay ${ }^{\mathrm{a}, \mathrm{b}, \mathrm{d}, *}$, F. Boschini ${ }^{\mathrm{a}, \mathrm{c}}$, R. Cloots ${ }^{\mathrm{a}, \mathrm{c}}, \mathrm{N}$. Vandewalle ${ }^{\mathrm{a}, \mathrm{b}}$ \\ a APTIS, Institute of Physics, University of Liege, Sart-Tilman, B-4000 Liège, Belgium \\ b GRASP, Institute of Physics, University of Liege, B-4000 Liège, Belgium \\ c LCIS-GreenMat, Institute of Chemistry, University of Liege, B-4000 Liège, Belgium \\ ${ }^{\mathrm{d}}$ F.R.S.-FRNS, B-1000 Bruxelles, Belgium
}

\section{A R T I C L E I N F O}

\section{Article history:}

Received 6 March 2012

Received in revised form 13 September 2012

Accepted 15 September 2012

Available online 23 September 2012

\section{Keywords:}

Powder characterization

Segregation

Separation

Granular flow

\begin{abstract}
A B S T R A C T
We propose a method using an original apparatus to amplify any segregation from a granular mixture. The device is a superposition of four Hele Shaw cells for producing a cascade of granular flows that enhances the segregation process. After the flow cascade, the concentration of each granular species is measured in the different cells. From these concentrations, a parameter $p$ that characterizes the ability of the mixture to segregate is calculated. The physical model used to calculate this parameter $p$ is presented. Finally, the method is validated with a series of experiments performed with common granular materials. Relevant parameters behind segregation are evidenced.
\end{abstract}

(c) 2012 Elsevier B.V. All rights reserved.

\section{Introduction}

Granular segregation is a counterintuitive phenomenon where an initially mixed state of dry particles separates into its components under excitation [1-6]. Although the phenomenon is known from a long time, its occurrence remains puzzling. Segregation is not only of fundamental interest but is also of practical importance with applications in areas ranging from the pharmaceutical industry to chemistry. The segregation process could be desirable in separation processes but in many cases, the segregation degrades the quality of the final product or causes safety problems. In order to avoid granular segregation in some industrial processes, two methods could be used. The first one is the optimization of the devices used to process the granular material $[7,8]$. The second one is the modification of the granular material itself. However, to change the granular material properties, the existence of a characterization method is the necessary condition. We address herein the fundamental and practical problems of measuring segregation from a granular mixture. For that purpose, we propose a method for amplifying the segregation phenomenon and we provide the definition of a segregation index $p$, measuring the ability of a mixture to separate into its components.

Granular segregation takes place when the grains have the possibility to rearrange in the granular random assembly. Kinetic energy is therefore needed and could be provided along different ways: horizontal or vertical shaking [3,9], granular flow [5,10-12], rotation

\footnotetext{
* Corresponding author at: GRASP, Institute of Physics, University of Liege, B-4000 Liège, Belgium.

E-mail address: geoffroy.lumay@ulg.ac.be (G. Lumay).
}

[13-16], air flow [17], ... Among others, a spectacular experiment has been proposed by Makse et al. [18,19]. In order to visualize the mechanisms, a Hele Shaw cell, which is a quasi two dimensional cell formed by two glass plates separated by a small gap, has been used. When grains are poured in a vertical Hele Shaw cell, a triangular heap builds up. This geometry has been extensively studied fundamentally [20-22]. Two segregation processes could be observed: (i) the larger grains are not trapped during the flow and reach the base of the pile, and (ii) the larger grains are going up in the flowing phase. The first process, called full segregation, leads to a clear separation of the pile where the small grains are on the top of the pile and the large grains are situated at the bottom. The second segregation process leads to a self-stratification. A combination of both patterns is observed in most cases. The conditions for the appearance of such patterns are associated to different packing properties of the granular species at rest and avalanching dynamics [23].

As quoted by Cizeau et al. [23], segregation taking place in a heap flow can be seen as originating from the different repose angles exhibited by the granular species. Indeed, by considering that the repose angle depends on the local composition of the surface, one defines $\theta_{\alpha \beta}$ as the generalized repose angle of a rolling grain of type $\alpha$ on a surface with local grains of type $\beta$. Different values of $\theta_{\alpha \beta}$ are expected when granular species are characterized by different sizes and shapes. Fig. 1 considers small spherical grains (type 1) and large irregular grains (type 2). One understands that the repose angle $\theta_{11}$ of the granular species 1 is small compared to the repose angle $\theta_{22}$ of the second species. Moreover, a small and spherical grain could be easily captured by a rough surface made of type 2 grains. The repose angle $\theta_{12}$ is therefore high. A large grain could however destabilize a layer of small spherical grains providing a low 

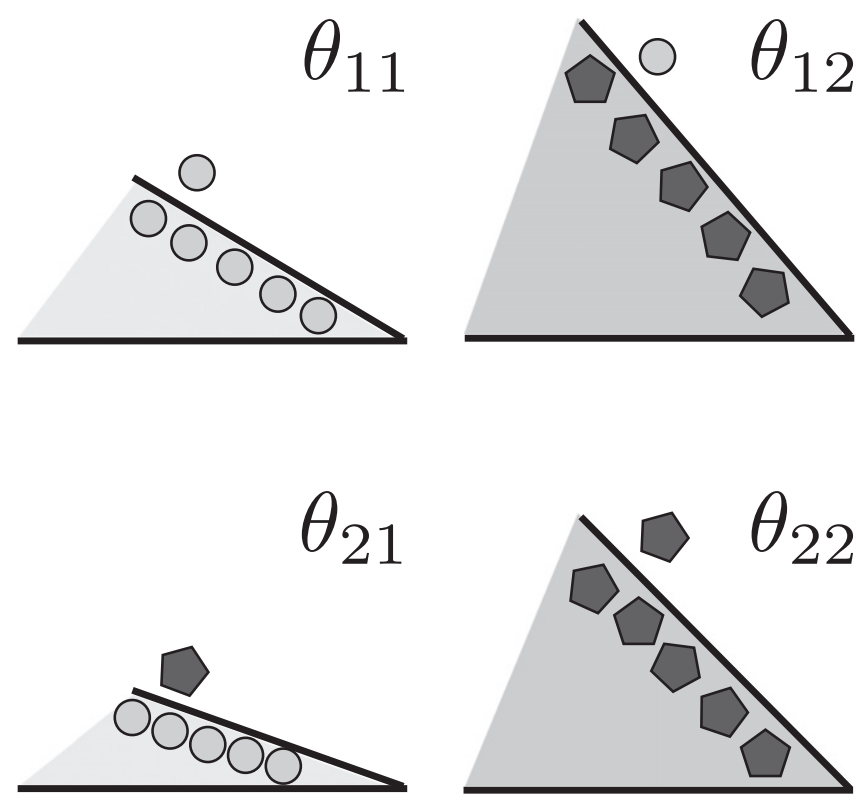

Fig. 1. A sketch of four dynamical angles of repose $\theta_{\alpha \beta}$ which depend on the composition of grains at the surface of the pile. Small spherical grains (1) and large irregular grains (2) are illustrated. As discussed in the text, $\theta_{11}<\theta_{22}, \theta_{12}>\theta_{11}$, and $\theta_{21}<\theta_{22}$.

$\theta_{21}$ value. Different values of $\theta_{\alpha \beta}$ imply that during heap formation, a specific granular species could be captured more frequently than the second one which rolls down. The four possible interactions between both species represent a key mechanism for segregation. An algorithm of heap formation based on four angles of repose $\theta_{\alpha \beta}$ is able to produce complex (and realistic) segregation patterns like selfstratification [23] or segregation "valleys" on irregular piles [24]. We should note that while the angles $\theta_{11}$ and $\theta_{22}$ are easy to determine experimentally, the angles $\theta_{12}$ and $\theta_{21}$ are difficult to estimate.

Many parameters are expected to influence the ability of a mixture to segregate: the grain size difference, the difference of grain density, the difference of grain shape and roughness, the cohesion [25] etc. The generalized repose angle $\theta_{\alpha \beta}$ includes all these parameters. The experimental device and the protocol presented hereafter can be used to study rigorously the influence of all the parameters separately. In this paper, we focus on the presentation of the device and a model is proposed to interpret the results. Finally, the system is validated with some typical studies. The effect of both repose angles $\theta_{11}$ and $\theta_{22}$ on the segregation is analyzed.

\section{Experiments}

After the formation of a heap in Hele Shaw cell (see Fig. 2), it is possible to collect the top part of the heap by opening an aperture at the left bottom corner of the triangular heap. Then, if the granular material has a tendency to segregate, the analysis of the obtained sample will show concentrations that differ from the initial values. The concentration differences could remain smaller than detection thresholds. That is the reason why we decided to elaborate a process for amplifying the segregation phenomena. The main idea is to submit the granular mixture to repeated flow cycles in order to separate the components, similarly to fractional distillation [26].

The proposed experimental setup consists in superposing four Hele Shaw cells for producing a flow cascade. Fig. 3 presents a sketch of the experimental set-up, as well as the various steps of the measurement cascade. The width and the thickness of the cells are respectively $100 \mathrm{~mm}$ and $15 \mathrm{~mm}$. Some pictures of the heaps obtained during a measurement are presented in Fig. 4. The top cell (named cell \#0) is a funnel that receives the granular mixture to be analyzed.

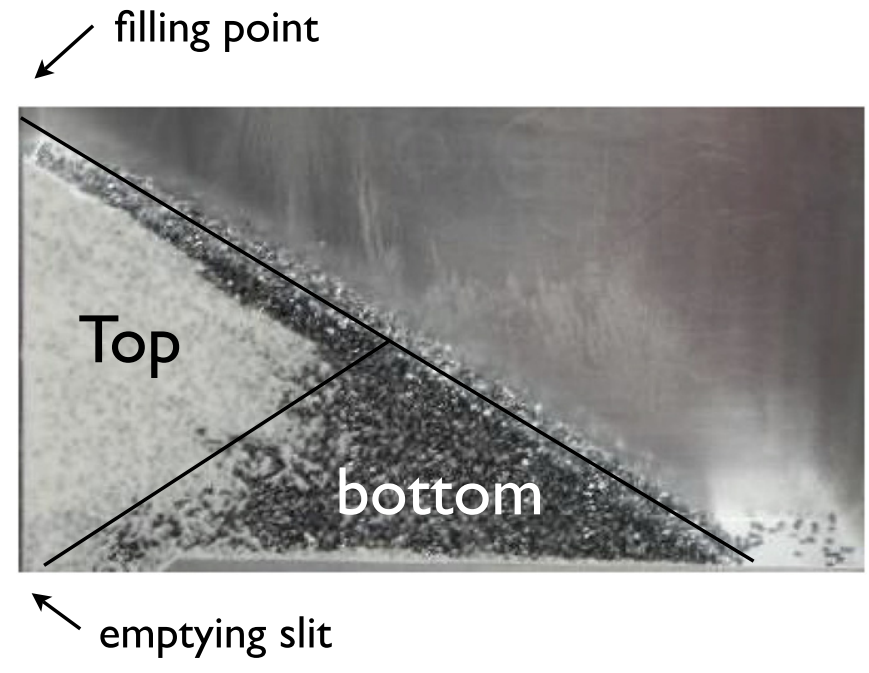

Fig. 2. Picture of a pile made of small (white) and big (black) grains in a Hele Shaw cell. The location of the filling point is indicated. By opening the slit situated at the left bottom corner of the cell, the top part of the pile could be extracted from the cell.

The mixture is made of a mass $m$ of one granular species and a mass $M$ of the other one. Consider $x_{0}=m / M$ being the ratio of both concentrations in the cell \#0. After pouring the grains in the top cell, the first aperture is opened and the grains flow in the cell \#1. The size of the aperture is $3 \mathrm{~mm}$ for all the measurements presented in this paper. During this flow, a segregation process may separate both granular components as shown by Fig. 4(a). The top of the obtained heap contains respectively a mass $p m$ of the first variety and a mass $(1-p) M$ of the second one. The dimensionless parameter $p$ represents the measure of heterogeneity induced by segregation. A value $p=1 / 2$ means homogeneity. Moreover, $p>1 / 2$ when $m$ and $M$ are corresponding respectively to the mass of small and large grains. The bottom of the pile will contain respectively a mass $(1-p) m$ of the first variety and a mass $p M$ of the second one. By opening the aperture below the cell \#1, the top part of the heap will flow in the cell \#2 (see Fig. 4(b)). Then, the concentration ratio of grains remaining in the cell \#1 is $x_{1}=(1-p) x_{0} / p$. Following the same reasoning, the top of the heap in cell \#2 contains a mass $p^{2} m$ of the first variety and a mass $(1-p)^{2} M$ of the second one. The bottom part of the heap in cell \#2 contains $(1-p) p m$ grains of the first variety and $(1-p) p M$ grains of the second one. Therefore, after the flow from the cell \#1 to the cell \#2, the concentration ratio remaining in the cell \#2 is $x_{2}=x_{0}$. Finally, after the opening of the last aperture, the top part of the heap of the cell \#2 falls in the cell \#3 (see Fig. 4(c)). Then, the concentration ratio in this last cell becomes $x_{3}=p^{2} /(1-p)^{2} x_{0}$. This model assumes that the segregation probability $p$ does not depend on the concentration of both species. The concordance between this model and the following experimental results will show that this approximation is good. By determining the concentrations of the granular components in the four cells, one has the following sequence:

$$
\left\{\begin{array}{l}
x_{0} \\
x_{1}=\frac{1-p}{p} x_{0} \\
x_{2}=x_{0}^{2} \\
x_{3}=\frac{p^{2}}{(1-p)^{2}} x_{0}
\end{array}\right.
$$

which allows to determine the segregation parameter $p$ from either $x_{1}$ or $x_{3}$. The parameter $p$, obtained from the concentration ratio $x_{3}$, takes advantage of the amplification process. Of course, the parameter extitp obtained from the ratio $x_{1}$ gives the same value but with a 

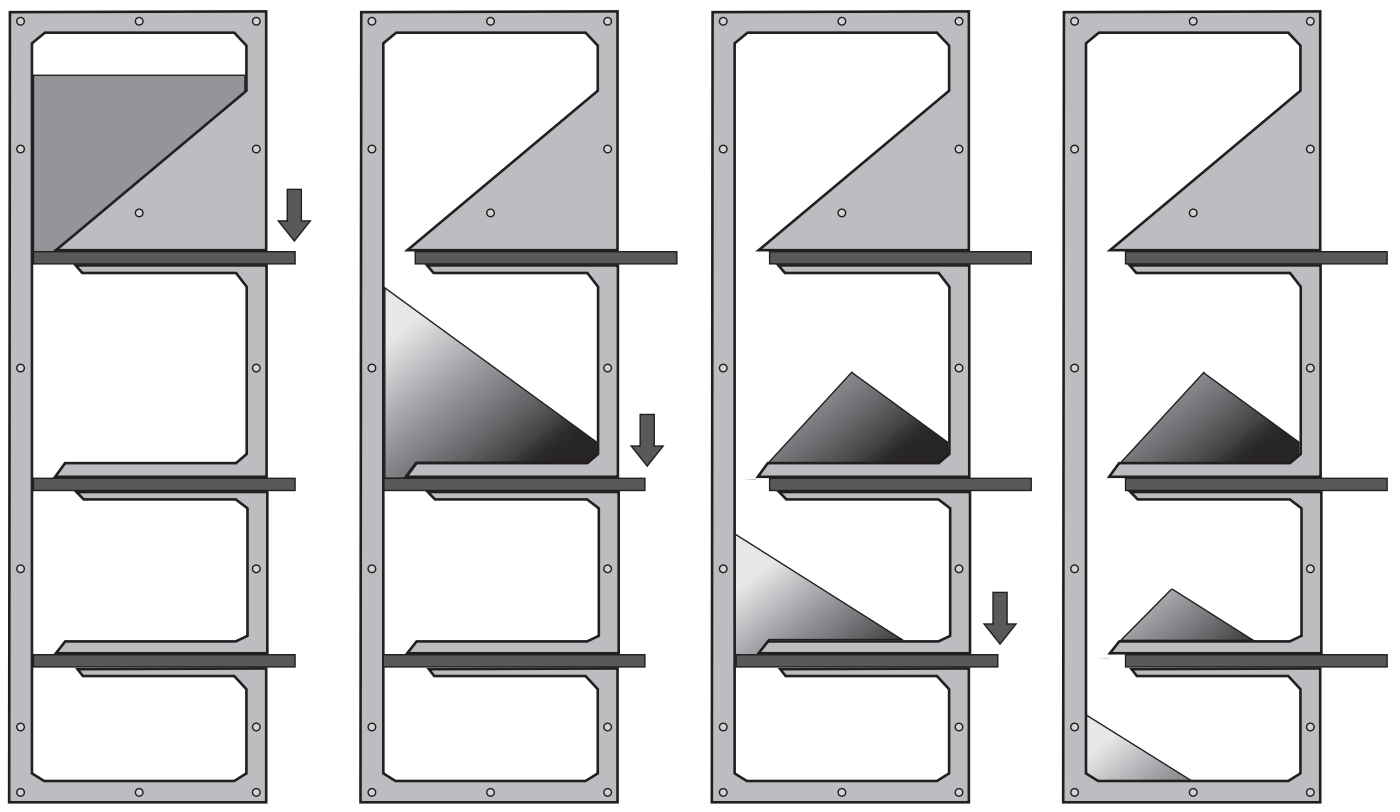

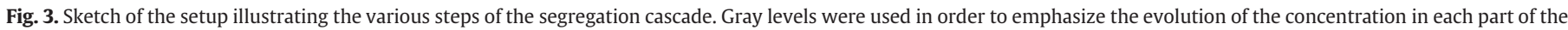
setup.

highest experimental uncertainty. In order to extract an accurate value of $p$, one could consider the 3 -degree polynom $f(n)$ given by

$f(n)=\alpha n^{3}+\beta n^{2}+\gamma n+1$

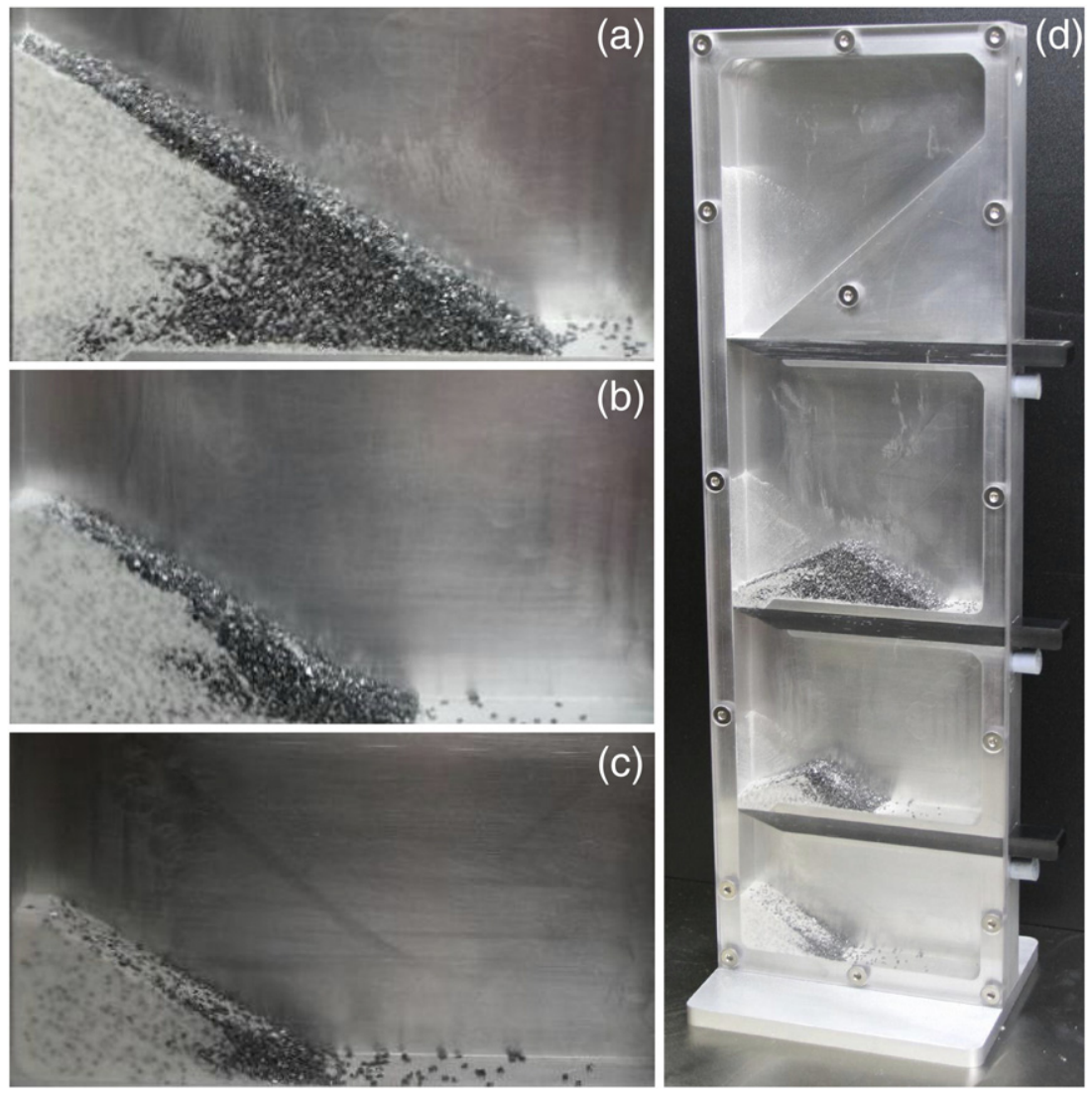

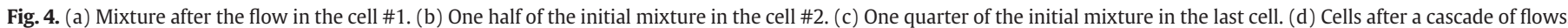
The width and the thickness of the cells are respectively $100 \mathrm{~mm}$ and $15 \mathrm{~mm}$. 
$\beta=\frac{(2 p-1)[(5 p-11) p+5]}{2 p(1-p)^{2}}$

$\gamma=\frac{1}{3(1-p)^{2}}-\frac{2}{3(1-p)}+\frac{3}{p}-6$

This function provides the values of $x_{n} / x_{0}$ given by Eq. (1). A fit of the normalized sequence $\left\{x_{1} / x_{0}, x_{2} / x_{0}, x_{3} / x_{0}\right\}$ by $f$ provides an accurate value for the single free parameter $p$. The segregation parameter obtained with this curve fitting method is noted as $p_{\text {fit }}$.

In order to validate our method, a series of measurements has been performed with binary mixtures made of four types of grains: spherical glass beads, silicon carbide abrasives, sugar and salt. The measurements have been performed with a relative humidity situated between $35 \%$ and $40 \%$ and a temperature of $20{ }^{\circ} \mathrm{C}$. The characteristics of the samples are summarized in Table 1. First, in order to validate the physical model, mixtures with two different grain sizes have been considered. The initial ratio is fixed to $x_{0}=1$. The ratios $x_{1}, x_{2}$ and $x_{3}$ are measured by sieving the samples extracted from the cells after the flow cascade. Fig. 5 presents these ratios as a function of the cell number. The amplification of the segregation process is clearly shown for three different mixtures. Moreover, as predicted by the model, we obtain $x_{1}<x_{0}, x_{2} \approx x_{0}$ and $x_{3}>x_{0}$. One should remark that small deviations from $x_{0}$ can be found for the ratio $x_{2}$ in the second cell. This could be attributed to non-linearities neglected in our approach. Fits using the polynom $f$ (see Eq. (2)) are shown. The agreement between measurements and idealized behavior in Eq. (1) is excellent, meaning that the definition of a segregation index is relevant.

The differences between the glass beads and the silicon carbide abrasives are essentially the grain size and roughness. The roughness has a strong impact on the repose angle $\theta$ of the granular material. This repose angle of the pure granular species has been measured with a GranuHeap instrument $[27,28]$. The results are presented in Table 1.

The values of the concentration ratios $\left(x_{1}, x_{2}\right.$ and $\left.x_{3}\right)$ and the corresponding segregation parameters $p$ and $p_{f i t}$ obtained for all the considered mixtures are presented in Table 2. The segregation parameters $p$ and $p_{f i t}$ have been obtained respectively with Eqs. (1) and (2). Both methods used to obtain the segregation probability are giving the same values for all the considered mixtures. The last column of Table 2 presents the error on $p_{\text {fit }}$ estimated by the LevenbergMarquardt fitting algorithm. The segregation parameter $p$ covers a wide range of values from 0.53 to 0.68 . The concentration ratios given in this table are the result of an average over three measurements. For binary mixtures of glass beads, the segregation parameter $p$ increases when the size difference between the components increases. A similar trend is observed for mixtures of glass bead and abrasive grain. Therefore, the grain size difference influences strongly the ability of the mixture to segregate. This result is in agreement with the theoretical and experimental work of Goyal et al. [21]. Indeed, they have shown that the concentration of small grains situated at the top of the pile increases when the grain size ratio increases. Moreover, with the glass beads and abrasive grain mixtures the

Table 1

Main characteristics of the 8 grain species used to validate the method and the physical model.

\begin{tabular}{lllll}
\hline Name & Type & Surface & Size & Repose angle \\
\hline Beads1 & Glass spheres & Smooth & $100 \mu \mathrm{m}$ & $31.3 \pm 0.5$ \\
Beads2 & Glass spheres & Smooth & $175 \mu \mathrm{m}$ & $23.7 \pm 0.5$ \\
Beads3 & Glass spheres & Smooth & $250 \mu \mathrm{m}$ & $23.3 \pm 0.5$ \\
Beads4 & Glass spheres & Smooth & $500 \mu \mathrm{m}$ & $20.3 \pm 0.5$ \\
GR80 & Silicon carbide & Rough & $170 \mu \mathrm{m}$ & $37.3 \pm 0.5$ \\
GR24 & Silicon carbide & Rough & $700 \mu \mathrm{m}$ & $39.2 \pm 0.5$ \\
Sugar & Sugar & Rough & $500 \mu \mathrm{m}$ & $40.5 \pm 0.5$ \\
Salt & Salt & Rough & $500 \mu \mathrm{m}$ & $35.5 \pm 0.5$ \\
\hline
\end{tabular}

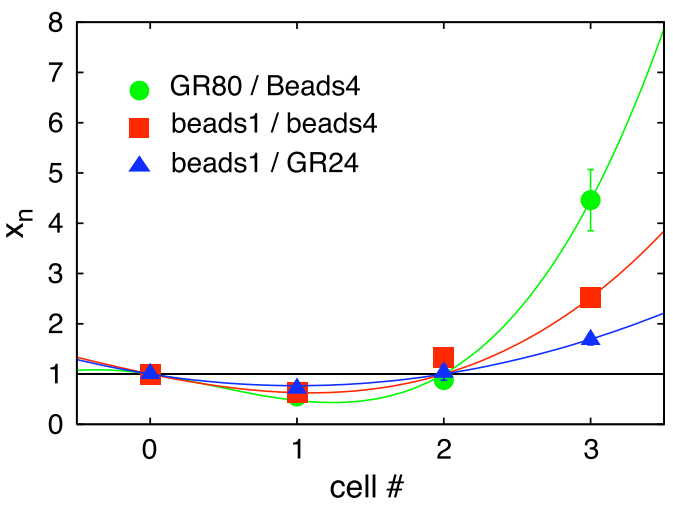

Fig. 5. Concentration ratio $x_{n}$ as a function of the cell number for three binary mixtures. The amplification of the segregation is clearly shown by the plot. The error bars are indicated. Curves are fits using Eq. (2) with a single free parameter $p$.

segregation parameter $p$ is different in the case of small glass/large abrasive and large glass/small abrasive. This result is in agreement with recent studies [22] showing that large rough grain and small smooth grain mixtures are forming stratification patterns while large smooth grains and small rough grains exhibit segregation. Therefore, the separation mechanisms are different. These results are showing that the repose angles $\theta_{11}$ and $\theta_{22}$ which are related to the difference in roughness characteristics of the grains (see Table 1 ) are a relevant parameter in the separation mechanism. This confirms results obtained in rotating tubes [29].

\section{Discussion}

As quoted in the introduction, segregation can be seen as originating from the four possible interactions between grain species as described by the angles $\theta_{\alpha \beta}$. Although simple algorithms produce realistic patterns, the major drawback of this concept is that only $\theta_{11}$ and $\theta_{22}$ can be measured experimentally. Those angles are given in Table 1 for selected grains. The angles $\theta_{12}$ and $\theta_{21}$ cannot be measured directly in experiments, even though they are relevant for describing segregation, as we will see below.

Let us consider Fig. 6 where the angle of repose is shown for each rolling phase as a function of the concentration $\phi$ of type 2. Extreme points correspond to the four angles defined in the introduction and illustrated in Fig. 1. As in [23], we choose for simplicity a linear behavior as a function of $\phi$ and the difference between both curves is a constant $\Psi=\theta_{11}-\theta_{21}=\theta_{12}-\theta_{22}$. This assumption is compatible with the observations made in the introduction and reduces the interaction matrix to three parameters $\left(\theta_{11}, \theta_{22}, \Psi\right)$ instead of four parameters.

\section{Table 2}

Values of the concentration ratios $\left(x_{1}, x_{2}\right.$ and $\left.x_{3}\right)$ and the corresponding segregation parameters $p$ and $p_{\text {fit }}$ obtained for all the considered mixtures. The measurements have been repeated three times and the presented ratios are the averaged values. The segregation parameters $p$ and $p_{f i t}$ have been obtained respectively with Eqs. (1) and (2). The last column presents the error on $p_{\text {fit }}$ estimated by the Levenberg-Marquardt fitting algorithm.

\begin{tabular}{llllllll}
\hline Species 1 & Species 2 & $x_{1}$ & $x_{2}$ & $x_{3}$ & $p$ & $p_{\text {fit }}$ & err \\
\hline Beads1 & Beads4 & 0.634 & 1.324 & 2.523 & 0.614 & 0.614 & 0.014 \\
Beads2 & Beads4 & 0.610 & 0.759 & 1.833 & 0.575 & 0.586 & 0.015 \\
Beads3 & Beads4 & 0.607 & 0.872 & 1.774 & 0.571 & 0.571 & 0.002 \\
GR80 & Beads4 & 0.555 & 0.882 & 4.460 & 0.679 & 0.661 & 0.013 \\
GR80 & GR24 & 0.716 & 0.949 & 1.816 & 0.574 & 0.577 & 0.005 \\
Beads1 & GR24 & 0.710 & 1.026 & 1.679 & 0.564 & 0.568 & 0.004 \\
Beads2 & GR24 & 0.811 & 0.832 & 1.276 & 0.530 & 0.531 & 0.009 \\
Beads3 & GR24 & 0.707 & 0.963 & 1.335 & 0.536 & 0.545 & 0.010 \\
Beads2 & Sugar & 0.639 & 0.731 & 1.723 & 0.568 & 0.570 & 0.015 \\
Beads2 & Salt & 0.643 & 0.838 & 1.657 & 0.563 & 0.571 & 0.012 \\
\hline
\end{tabular}




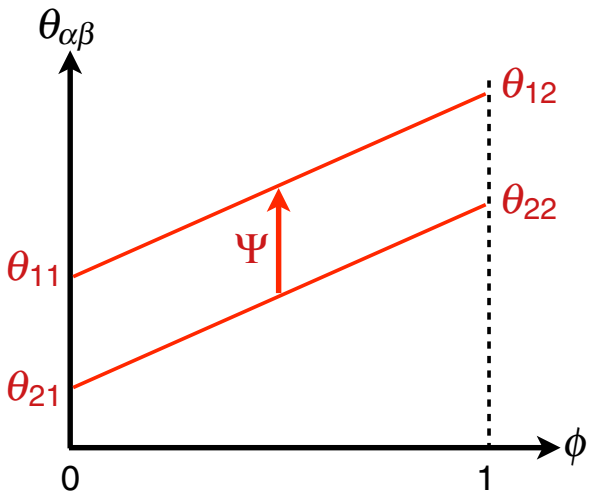

Fig. 6. The repose angle for two types of rolling grains as a function of the concentration of surface grains $\phi$ of type 2. The four angles illustrated on Fig. 1 are the angles found for respectively $\phi=0$ and $\phi=1$. As in [23], the repose angle difference is considered as a constant $\Psi$.

Whatever the concentration $\phi$, a grain of type 1 has a high probability $P_{1}$ to be captured by the surface grain layer compared with the probability $P_{2}=1-P_{1}$ for the second grain species. This is due to the difference of repose angles as presented on the sketch of Fig. 6 . When a grain is rolling along the free surface, it visits numerous different local configurations before being captured. Although the system is out of equilibrium, the configuration space is visited by the system and one could propose a simple (and classical) expression

$P_{1}=\frac{\exp (\Psi)}{\exp (\Psi)+\exp (-\Psi)}$

which considers only the difference $\Psi$, being the "barrier" to be overcome for trapping the second grain species. One has indeed a probability

$P_{2}=\frac{\exp (-\Psi)}{\exp (\Psi)+\exp (-\Psi)}$

One recovers $P_{1}=P_{2}=1 / 2$ when the repose angles are identical $(\Psi=0)$. By considering that our parameter $p$ is linked to capture events during the granular flow $\left(p=P_{1}\right)$, the combination of the above equations gives

$\ln \left(\frac{p}{1-p}\right)=2 \Psi$

meaning that any measure of the parameter $p$ provides an indirect estimate of the parameter $\Psi$. Our method is therefore relevant for any modeling of segregation because the dimensionless parameter $p$ could be linked to intrinsic properties of the granular species. Further theoretical work is of course needed for elucidating this point but this remains outside the scope of the present paper.

\section{Conclusion}

We developed a protocol dedicated to the quantification of the granular segregation in a granular mixture. It is based on an amplification process based on a cascade of granular flows. The method can be easily implemented and gives reliable and interpretable results. Mixtures of common granular materials have been analyzed in order to illustrate and to validate this experimental approach. The effect of both repose angles $\theta_{11}$ and $\theta_{22}$ on the segregation processes is evidenced. A segregation index $p$ is defined. We have shown how it can be related to earlier theoretical works. Then, the proposed method is a link between recent fundamental works on granular segregation and the need of a practical segregation characterization method.

\section{Acknowledgments}

We thank S. Rondia for the CNC milling of the segregation cell. GL thanks F.R.S.-FNRS. This work has been also supported by the INANOMAT project (IAP P6/17) of the Belgian Science Policy.

\section{References}

[1] L. Bates, User Guide to Segregation, British Materials Handling Board, 1997.

[2] T. Shinbrot, F. Muzzio, Noise to order, Nature 410 (2001) 251.

[3] A. Kudrolli, Size separation in vibrated granular materials, Reports on Progress in Physics 67 (2004) 209.

[4] J. Duran, J. Rajchenbach, E. Clément, Arching effect model for particle size segregation, Physical Review Letters 70 (1993) 2431.

[5] N. Lecocq, N. Vandewalle, Stripes ordering in self-stratification experiments of binary and ternary granular mixtures, Physical Review E 62 (2000) 8241.

[6] K. Johanson, C. Eckert, D. Ghose, M. Djomlija, M. Hubert, Quantitative measurement of particle segregation mechanisms, Powder Technology 159 (2005) 1.

[7] P. Tang, V.M. Puri, Methods for minimizing segregation, Particulate Science and Technology 22 (2004) 321.

[8] J.J. McCarthy, Turning the corner in segregation, Powder Technology 192 (2009) 137.

[9] M.J. Metzger, B. Remy, B.J. Glasser, All the Brazil nuts are not on top: vibration induced granular size segregation of binary, ternary and multi-sized mixtures, Powder Technology 205 (2011) 42.

[10] A. Samadani, A. Pradhan, A. Kudrolli, Size segregation of granular matter in silo discharges, Physical Review E 60 (1999) 7203.

[11] N. Engblom, H. Saxen, R. Zevenhoven, H. Nylander, G.G. Enstad, Segregation of powder mixtures at filling and complete discharge of silos, Powder Technology 215 (2012) 104

[12] W.R. Ketterhagen, J.S. Curtis, C.R. Wassgren, B.C. Hancock, Modeling granular segregation in flow from quasi-three-dimensional, wedge-shaped hoppers, Powder Technology 179 (2008) 126.

[13] S.W. Meier, D.A.M. Barreiro, J.M. Ottino, R.M. Lueptow, Coarsening of granular segregation patterns in quasi-two-dimensional tumblers, Nature Physics 4 (2008) 244.

[14] I. Zuriguel, J.M.N.T. Gray, J. Peixinho, T. Mullin, Pattern selection by a granular wave in a rotating drum, Physical Review E 73 (2006) 061302.

[15] I. Zuriguel, J. Peixinho, T. Mullin, Segregation pattern competition in a thin rotating drum, Physical Review E 79 (2009) 051303.

[16] F. Cantelaube, D. Bideau, S. Roux, Kinetics of segregation of granular media in a two-dimensional rotating drum, Powder Technology 93 (1997) 1

[17] V. Sibanda, R.W. Greenwood, J.P.K. Seville, Y. Ding, S. Iyuke, Predicting particle segregation in cross-flow gas filtration, Powder Technology 203 (2010) 419.

[18] H.A. Makse, S. Havlin, P.R. King, H.E. Stanley, Spontaneous stratification in granular mixtures, Nature 386 (1997) 379-381.

[19] H.A. Makse, R.C. Ball, H.E. Stanley, S. Warr, Possible stratification mechanism in granular mixtures, Physical Review E 58 (1998) 3357-3368.

[20] J. Baxter, U. Tüzün, D. Heyes, I. Hayati, P. Fredlund, Stratification in poured granular heaps, Nature 391 (1998) 136

[21] R.K. Goyal, M. Silvina Tomassone, Power-law and exponential segregation in two-dimensional silos of granular mixtures, Physical Review E 74 (2006) 051301

[22] M. Shimokawa, S. Ohta, Spontaneous formation of dual stratification patterns in a large quasi-two-dimensional sand pile, Physical Review E 77 (2008) 011305.

[23] P. Cizeau, H.A. Makse, H.E. Stanley, Mechanisms of granular spontaneous stratification and segregation in two-dimensional silos, Physical Review E 59 (1999) 4408.

[24] N. Vandewalle, R. DÕhulst, M. Ausloos, Phase segregation in binary sandpiles on fractal bases, Physical Review E 59 (1999) 631.

[25] A. Samadani, A. Kudrolli, Segregation transitions in wet granular matter, Physical Review Letters 85 (2000) 5102.

[26] R.H. Perry, D.W. Green, in: Perry's Chemical Engineers' Handbook, McGraw-Hill, 2007.

[27] GranuHeap instrument is described on www.aptis.be.

[28] G. Lumay, F. Boschini, K. Traina, S. Bontempi, J.C. Remy, R. Cloots and N Vandewalle, Measuring the flowing properties of powders and grains, accepted for publication in, Powder Technology (2012).

[29] H. Caps, R. Michel, N. Lecocq, N. Vandewalle, Long lasting instabilities in granular mixtures, Physica A 326 (2003) 313. 